AperTO - Archivio Istituzionale Open Access dell'Università di Torino

\title{
Ranking alien species based on their risks of causing environmental impacts: A global assessment of alien ungulates
}

\section{This is the author's manuscript}

Original Citation:

Availability:

This version is available http://hdl.handle.net/2318/1765739

since 2022-01-24T14:49:48Z

Published version:

DOI:10.1111/gcb.15467

Terms of use:

Open Access

Anyone can freely access the full text of works made available as "Open Access". Works made available under a Creative Commons license can be used according to the terms and conditions of said license. Use of all other works requires consent of the right holder (author or publisher) if not exempted from copyright protection by the applicable law. 
Volery, L., Jatavallabhula, D., Scillitani, L., Bertolino, S., \& Bacher, S. (2020). Ranking alien species based on their risks of causing environmental impacts: a global assessment of alien ungulates. Global Change Biology, doi.org/10.1111/gcb.15467

\title{
Ranking alien species based on their risks of causing environmental impacts: a global assessment of alien ungulates
}

\author{
Volery Lara ${ }^{1}$, Jatavallabhula Divija ${ }^{1}$, Scillitani Laura ${ }^{2}$, Bertolino Sandro ${ }^{2}$, Bacher Sven ${ }^{1}$ \\ ${ }^{1}$ Department of Biology, University of Fribourg, Chemin du Musée 10, CH-1700 Fribourg, Switzerland \\ ${ }^{2}$ Department of Life Sciences and Systems Biology, University of Turin, Via Accademia Albertina 13, 10123 Torino, Italy
}

\begin{abstract}
For an efficient allocation of the limited resources to alien species management, the most damaging species should be prioritized. Comparing alien species based on their impacts is not straightforward, as the same species can cause different types and magnitudes of impacts when introduced to different contexts, making it difficult to summarize its overall impact. The Environmental Impact Classification of Alien Taxa (EICAT) systematically summarizes and compares detrimental impacts caused by alien populations to native biota and has been adopted by the International Union for Conservation of Nature (IUCN). For each alien species, all reported impacts to native populations within the introduced range are classified into five levels of severity, from negligible impact to irreversible local extinction. Currently, EICAT only compares alien species based on their highest impact, thereby ignoring variation in impact magnitudes. Here, we used information on the variation in impact magnitudes of alien species to estimate their risks to cause high impacts if introduced to a novel environment. We demonstrate the usefulness of this approach by classifying the global impacts of alien ungulates. We found impact reports for 27 of the 66 alien ungulate species established worldwide, highlighting substantial knowledge gaps in invasion science. We classified a total of 441 impacts to native fauna and flora caused by these 27 species. Twenty-six of the species were found to cause harmful impacts (native population declines or local extinctions). Mouflon (Ovis orientalis, Gmelin, 1774) and dromedary (Camelus dromedarius, Linnaeus, 1758) had a higher risk of causing local extinctions if introduced to a novel environment than sika deer (Cervus nippon, Temminck, 1838) and goats (Capra hircus, Linnaeus, 1758). Including risk of high impacts allows to discriminate among species with the same EICAT classification and improves alien species prioritization for management.
\end{abstract}

Keywords: IUCN, EICAT assessments, invasive species, introduced species, impact classification

Running title: Impact risks of alien ungulates

\section{Introduction}

Alien species introductions are accumulating around the globe at increasing and concerning rates (Seebens et al., 2017). Though the majority of alien species are not perceived as harmful, occasionally they can cause serious impacts to their recipient environments, leading to irreversible changes, such as causing local or global species extinctions (Bellard, Cassey, \& Blackburn, 2016; Pyšek et al., 2017). In order to effectively allocate the limited resources available to alien species management, identifying the most damaging species for prioritization is critical (Roy et al., 2014, 2015). This is not straightforward because impacts can occur in different environments and through various mechanisms, and because the same species might cause different types and magnitudes of impacts when introduced to different contexts (Parker et al., 1999; Ricciardi et al., 2013), making it difficult to summarize the overall impact of an alien species in a meaningful way and thus to compare species. 
The first challenge is to compare the various changes alien populations are causing in their recipient environments (Nentwig, Kühnel, \& Bacher, 2010). The Environmental Impact Classification for Alien Taxa (EICAT) allows the classification of impacts in a standardized way, making comparisons among diverse taxa and impact scenarios possible (Blackburn et al., 2014; Hawkins et al., 2015; IUCN, 2020a, 2020b): impacts are defined as detrimental effects on local native populations and are classified into five magnitudes (from Minimal Concern to Massive), depending on which level of organisation of the native populations is affected (decreased performance of individuals, population decline, or local extinction; Table 1). EICAT can be applied to the impacts caused by any alien taxon, in any type of environment, and through any mechanism. The criteria used in EICAT for classifying impacts are independent of subjective value judgements and only rely on empirical evidence (i.e. direct observations of impact). EICAT was recently adopted by the International Union for Conservation of Nature (IUCN) as its formal classification system of alien species' impacts (IUCN, 2020b). So far, EICAT has been implemented to classify the impacts of alien birds (Evans, Kumschick, \& Blackburn, 2016) and amphibians (Kumschick et al., 2017), marine fishes invasive to the Mediterranean (Galanidi, Zenetos, \& Bacher, 2018), alien bamboos (Canavan, Kumschick, Roux, Richardson, \& Wilson, 2019), gastropods alien to South Africa (Kesner \& Kumschick, 2018), alien terrestrial invertebrates in the pet trade of South Africa (Nelufule et al., 2020) and feral mammals in South Africa (Hagen \& Kumschick, 2018).

Under EICAT, alien species are classified and compared according to their highest recorded impact magnitude (Hawkins et al., 2015; IUCN, 2020b). Using only the maximum impact to classify alien species results in five coarse classifications. This might be uninformative when applied to larger groups of alien species (e.g. for global IUCN assessments of major taxa) because many aliens will receive the same rank. It also means that a large part of the available information about an alien's impact is ignored in the ranking, in particular the variation in impact magnitudes. For example, an alien species that has consistently been causing high impacts to native species in a variety of environmental contexts and another alien species having only occasionally caused high impacts, under very specific environmental conditions, would be classified the same under EICAT. However, it seems plausible that the former alien presents a higher risk of causing high impacts if introduced in a new place than does the latter. Since historical records of impacts can be used as a predictor of an alien's future impacts (Kulhanek, Ricciardi, \& Leung, 2011; Ricciardi, 2003), we propose that the frequency at which a species' introduced populations caused its highest impact magnitude could be incorporated in EICAT assessments as an indicator of the alien's risk of causing high impacts when introduced. This would allow more fine-grained comparisons among alien species: aliens with greater risks of causing high impacts could be prioritized for management over aliens with lower risks.

Here, we present a procedure to incorporate the risk of alien species to cause their highest impact into global EICAT assessments. We demonstrate the usefulness of our approach by systematically reviewing and classifying the impacts caused by alien ungulates worldwide using EICAT. Ungulates have been extensively introduced over the world in various regions and environments for farming and hunting purposes, making them an ideal group for capturing and investigating impact variation (Long, 2003; Spear \& Chown, 2009). We compare the ranking of alien ungulates by only considering their highest impact (current EICAT procedure) with rankings obtained by additionally considering the risk of the species to cause these highest impacts (new procedure). By estimating the risks of alien species to harm native biota through different mechanisms, this study represents a first step towards more meaningful predictions of their impacts across taxa.

In addition, we used our EICAT assessments to investigate factors associated with high impacts and biases in impact reporting. The EICAT classification allows the synthesis of available knowledge on alien species' impacts: it is therefore subject to the biases existing in invasion science. Geographic and taxonomic information biases have been already been identified in the field (e.g. Pyšek et al., 2008; Hulme et al., 2013; Evans and Blackburn, 2020). Measey et al. (2020) recently showed that studies on high impacts (i.e. impacts involving local extinctions of native populations) require more complex designs and are more costly; this might be introducing another bias, where easy-to-demonstrate impacts are more studied because of their lower cost and complexity. On the other hand, negligible impacts are likely to be under-represented compared to higher impact magnitudes, as most studies aim at reporting impacts instead of the absence of impacts. It should also be noted that although the criteria of the five EICAT impact magnitudes are independent of subjective judgement, the assessment process is not: the assessor, by translating impact observations into one of the five EICAT category, might incorporate some biases as well (González-Moreno et al., 2019). However, 
this bias may be minimised by exchange between assessors and assessments' reviews by independent assessors (González-Moreno et al., 2019).

\section{Material \& Methods}

Ungulate species with alien populations

A list of 66 ungulate species (orders Cetartiodactyla, Perissodactyla and Proboscidea), from 6 families, with established alien populations was compiled based on the Global Register of Introduced and Invasive Species (GRIIS) database (http://www.griis.org; accessed in March 2017). Proboscidea are closely related to ungulates and were included because of their functional similarity. In cases of taxonomic ambiguity (e.g. deciding whether a taxon is a distinct species or a sub-species), we followed the taxonomy of the IUCN Red List of Threatened Species (hereafter 'IUCN Red List'; https://www.iucnredlist.org). Re-introductions, introductions of hybrid populations (e.g. the introduction of Bison bison x Bison bonasus hybrids in the Caucasus mountains; Zablotskaya et al., 2004) and introductions of nonnative subspecies were not considered.

\section{Comparing impacts caused by alien species: EICAT assessments}

For each of the 66 ungulate species with alien populations, we performed a search for (peer-reviewed and grey) literature reporting observations of negative impacts caused by their established alien populations on native populations (i.e. primary sources). We followed the search protocol described in Evans et al. (2016): search terms (e.g. 'introduced' OR 'invasive' OR 'alien' OR 'non-native' OR 'non-indigenous' OR 'feral' OR 'exotic' AND 'sika deer' OR 'Cervus nippon') were used to find impact reports in online databases (Google Scholar [https://scholar.google.com], Web of Science [https://webofknowledge.com], the CABI's Invasive Species Compendium [ISC; https://www.cabi.org/ISC] and Google [https://www.google.com]), until no new information sources were found. Impact reports containing relevant information for EICAT assessments were selected based on the title, abstract and a screening of the content. We did not assess impact observations described in secondary sources (e.g. reviews) but always search for primary reports (but see Sheet 1 in Supp. 2, for inaccessible primary reports). In accordance with the EICAT standards (IUCN, 2020b; Volery et al., 2020), only observed impacts were classified; potential, hypothetical, projected or extrapolated impacts were considered non-relevant (but see Sheet 2 in Supp. 2, for non-relevant information sources). Species for which no impact observation was found were classified as Data Deficient (IUCN, 2020a).

We classified 441 impact observations on native biota into one of the five EICAT magnitudes (Table 1), based on the EICAT guidelines (IUCN, 2020b, 2020a). Each impact observation was also assigned to one of 12 impact mechanisms (competition, predation, hybridisation, transmission of disease, parasitism, poisoning/toxicity, bio-fouling or other direct physical disturbance, grazing/herbivory/browsing, chemical/physical/structural impact on ecosystem, indirect impact through interaction with other species), which can be grouped into direct (i.e. alien taxon directly interacts with the impacted native taxon) and indirect (i.e. alien taxon modifies another factor of the environment, thereby indirectly affecting the native taxon) mechanisms (Volery et al., 2020). Uncertainty was captured by assigning a confidence level (high, medium or low) to each observation indicating how confident the assessor is that the assigned magnitude is the 'true' one (IUCN, 2020a; Volery et al., 2020). A High confidence level indicates that the assessor is confident that the assigned magnitude is the true one, a Medium confidence level indicates that there is potential for the true magnitude to be different from the assigned one, and a Low confidence level indicates that it is likely that the true magnitude is different from the assigned one. More details on the assessment procedure are given in Supp. 1a.

Each impact of a certain magnitude, associated with a confidence level, and occurring through a particular mechanism at a specific location and time (affecting one or more native species) was considered as an impact observation. For each impact observation, the following information was collected: reference of the report, quotation (extracted from the report by the assessor and used as the rationale for the assigned impact magnitude), impact magnitude, impact mechanism(s) (and type: direct vs indirect), confidence score, confidence score rationales, impacted native species (and kingdom), location of impact (precise location, region [i.e. country's sub-unit such as district, state, territory, county, etc. or island/archipelago], country, sub-continent, and continent), assessor ID, date of assessment, and reviewer ID. To minimise assessor biases (González-Moreno et al., 2019), all classifications of impact magnitude, 
mechanism and confidence score were reviewed by at least one independent expert. Discrepancies between the assessor and the reviewer were explained and a consensus score was reached after discussion. This is similar to the IDEA protocol for structured expert elicitation (Hemming et al., 2018), but differs in that discussion of scores was not anonymous and that consensus scores were reached (i.e. all assessors agreed on a score). All assessments and reviews were carried out by DJ, LV and LS.

\section{Patterns in impacts and potential biases}

All statistical analyses were performed in $\mathrm{R}$ version 3.6.2 ( $\mathrm{R}$ Core Team, 2019). We first tested whether indirect or direct mechanisms led to higher impacts, we grouped harmful (MO, MR and MV) against non-harmful (MC and MN) impacts (IUCN, 2020b; Table 1) and tested for an association using a Generalized Linear Mixed-Effects Model (GLMM), with region of impact as random factor (glmer function of the Ime4 package; version 1.1-21, Bates et al., 2014) to account for spatial autocorrelation. We tested this on all 441 impact observations (excluding observations occurring through both direct and indirect mechanisms) and we assumed that the response variable (harmful/lower impacts) followed a binomial distribution. To test whether native flora or fauna was more severely affected by alien ungulates, we again grouped harmful against non-harmful impacts and used a GLMM, with region of impact as a random factor. Mechanism type (direct/indirect) and impacted kingdom (flora/fauna) are correlated, therefore we tested them separately.

To further investigate patterns in impacts and potential information biases, we compiled a list of the countries to which each ungulate species has been successfully introduced (see Tables S1, S2 and S3 in Supp. 1 for summaries, and Sheet 3 in Supp. 2 for the complete list), based on Long (2003), the ISC (accessed in November 2020), the IUCN Red List (accessed in November 2020), the Global Biodiversity Information Facility (GBIF, https://www.gbif.org; accessed in November 2020), GRIIS (accessed in 2017), and our own EICAT assessments. We used a Linear Mixed-Effects Model (Imer function of the Ime4 package) to test if the number of impact observations per country increased with the number of ungulate species introduced to that country, with continent as a random effect. We tested this on all countries with available impact observations, as well as on countries with no impact observations but with at least six introduced ungulate species (see Table S2 in Supp. 1). Both variables were log-transformed and the predictor variable was scaled to 0 mean and 1 standard deviation.

We used a simple linear model (Im function) to test if the number of impact observations of a species increased with the number of countries the species has been introduced to. We used data on all 66 ungulate species; species classified as Data Deficient were assigned as having 0 impact observations. Both variables were log-transformed and the predictor variable was scaled to 0 mean and 1 standard deviation. Finally, we used a simple linear model to test whether species causing higher impacts were more studied (log-transformed number of impact observations), and a generalized linear model ( $\mathrm{g} / \mathrm{m}$ function) to test whether more widely introduced species (log-transformed numbers of countries each species has been introduced to) were causing higher impacts. For both tests, we excluded Data Deficient species and used the highest impact magnitudes for the assessed species.

Distributions of residuals for all fitted (Generalized) Linear Mixed-Effects Models were interpreted using the testDispersion and simulateResiduals functions of the DHARMa package (version 0.3.3.0; Hartig, 2020; with default number $[n=250]$ of simulations).

Comparisons of the frequency distributions of the confidence scores across impact magnitudes (harmful/lower impacts) were conducted using a Pearson's Chi-squared test (chisq.test function). To compare the distributions of impact magnitudes (harmful/lower impacts) across assessed vertebrate groups (ungulates, amphibians [Kumschick et al., 2017] and birds [Evans et al., 2016]), we used an unconditional exact functional test (small expected values) (fun.chisq.test function of the FunChisq package; version 2.4.9.2, Zhong \& Song, 2019).

Comparisons between alien species within impact categories: Incorporating risk to comparisons between species

We first classified all species into the impact categories based on their highest impact magnitude following EICAT guidelines (IUCN, 2020b). 
Within each EICAT impact category, we aimed to distinguish between alien species that systematically cause their highest impact magnitude when introduced to a novel environment from alien species that only occasionally cause their highest impact magnitude. Only considering the frequency at which the species' populations caused their highest impacts (e.g. 1 out of 10 introduced populations) does not account for differences in 'sampling effort' between species. For example, a species widely introduced and having caused harmful impacts every time it has been introduced would not be differentiated from a species introduced once and having caused harmful impacts. However, we can be more confident of the high risk to cause harmful impacts for the widely introduced species than for the species introduced once. The more often a species has been (successfully) introduced and studied, the more information we have on the variation in its impact magnitude and thus on its risk to cause harmful impacts when introduced. To account for these differences in 'sampling effort' among species, we calculated Bayesian binomial $95 \%$ confidence intervals (Cls) and their highest probability density (hpd) means from the frequencies at which the species' populations caused their highest impacts, using the function binom. bayes of the R package binom (version 1.1-1; Sundar, 2014) with a flat beta prior distribution $(\alpha=\beta=1)$. The hpd means were used as estimations of the species' risk of causing their highest impact magnitude when introduced (hereafter 'impact risk'). We used, for each species, the number of regions (i.e. countries' sub-units; 'region' in our EICAT dataset) with impact observations as a proxy of the number of studied introduced populations. Species with few studied regions (i.e. species introduced to few regions and/or poorly studied species) will have wide Cls, providing limited information about their impact risks, whereas widely introduced and studied species will have narrower Cls. Using the number of studied regions allowed to account for differences in country sizes.

The species' impact risks were used to rank ungulate species from the most to least detrimental within their impact category (i.e. from the one with the highest to the lowest impact risk). To test whether the impact risks of the species classified in the same impact category significantly differed from each other, we performed pairwise comparisons of their impact risks' Cls (generated based 100,000 simulations from the frequencies at which each species caused its highest impact magnitude, by using the function rbeta of the binom package). Two Cls with an overlap of $<10 \%$ were considered significantly different from each other (the R code for this significance test is provided in Supp. 1b).

\section{Results}

\section{Comparing impacts caused by alien species: EICAT assessments}

We found 281 reports documenting 441 impact observations for 27 of the 66 ungulate species with populations introduced outside their native range (Table 2; see Sheet 4 of Supp. 2 for the complete database). The remaining 39 species were classified as Data Deficient (Table S1 in Supp. 1). In addition, we recorded 252 inaccessible primary reports and 436 non-relevant reports (e.g. reports describing potential impacts, reports describing impacts on soil properties, etc.; see Sheets 1 and 2, Supp. 2).

Impact observations of alien ungulates were most often assigned Moderate (native population decline; $46 \%$ ) or Minor (decrease in performance of native individuals; $44 \%$ ) magnitudes (Fig. 1; Table S4 in Supp. 1). Eight species, the Javan deer (Rusa timorensis, de Blainville, 1822), dromedary, mouflon, cattle (Bos taurus, Linnaeus, 1758), sika deer, sheep (Ovis aries, Linnaeus, 1758), white-tailed deer (Odocoileus virginianus, Zimmermann, 1780) and goat, caused extirpations of at least one local native population (Major impacts). All alien ungulates at least once caused a decline in a native population (Moderate impact) (Table 1), except for the American bison (Bison bison, Linnaeus, 1758), which was only documented to cause one Minor impact. $50 \%$ impact observations were assigned a Low confidence score, $42 \%$ a Medium confidence score and the remaining $8 \%$ observations were assigned a High confidence score (Fig. 1; Table S5 in Supp. 1). Confidence scores were equally distributed across impact magnitudes ( $p=0.23$; Table S6 in Supp. 1).

Impacts of alien ungulates were caused through 8 mechanisms (Fig. 2; Table S7 in Supp. 1): the most frequently recorded mechanisms were grazing/herbivory/browsing (59 \%), direct physical disturbance (18\%), and chemical/physical/structural impact on ecosystems (15\%). Sika deer was the only species with reported impacts through hybridization in the wild, and goats and wild boars (Sus scrofa, Linnaeus, 1758) were the only species found 
to cause impacts through predation. Local extirpations of native populations (Major impacts) were only reported through grazing/herbivory/browsing (43\%), hybridization (36\%) and chemical/physical/structural impacts on ecosystems (21\%). Native population declines (Moderate impacts) occurred through all 8 mechanisms but mainly through grazing/herbivory/browsing (55\%), chemical/physical/structural impacts on ecosystems ( $25 \%)$ and direct physical disturbance (e.g. trampling; $18 \%$ ). Indirect mechanisms (competition, transmission of disease, chemical/physical/structural impact on ecosystem, indirect impact through interaction with other species) were recorded less frequently than direct mechanisms (predation, hybridisation, direct physical disturbance, grazing/herbivory/browsing) ( $20 \%$ vs. $80 \%$ ), but led to higher impact magnitudes ( $p<0.001$; Table S8 in Supp. 1). Except for seven impact observations, plants were only affected via direct mechanisms, whereas animals were mostly (64\%) indirectly affected. Native plants were affected more than twice as often as native animals (70\% vs. $30 \%$; Table S9 in Supp. 1), but animals were affected more severely than plants ( $p=0.004$; Table S10 in Supp. 1). However, we found both native plants and native animals to have suffered local extirpations (Major impacts).

For most indirect mechanisms, we found more reports describing the possibility that alien species cause impacts or describing impact mechanisms than reports describing impact magnitudes. For instance, for the mechanism competition, we found 60 sources showing diet or niche overlap (see Sheet 2 in Supp. 2), which were not used in the EICAT classification, but only 18 direct observations of competition impacts (classified with EICAT). Likewise, for transmission of disease, 20 articles showed that alien or native individuals were hosts of parasites, but only 4 studies reported how much native individuals or populations were suffering from the infection (see Sheet 2 in Supp. 2). Several articles described alien ungulates as dispersal agents of alien plants or fungi (e.g. Davis, Forsyth, \& Coulson, 2010; Loydi \& Zalba, 2009; O'Connor \& Kelly, 2012), but the consequences of this for native species were not measured, preventing their classification under EICAT. By contrast, this was not the case for the indirect mechanism 'chemical/physical/structural impact on ecosystems', for which impact magnitudes were often studied (in 68 impact observations).

Impact observations of alien ungulates were recorded in 34 countries on seven continents, but mainly in the United States (27\%), New Zealand (14\%), the United Kingdom (11\%), Australia (11\%), Canada (9\%) and Argentina (5\%) (Fig. 3; Table S2 in Supp. 1). Although we found the number of impact observations for a country to generally increase with the number of introduced ungulate species (Table S11 in Supp. 1), we identified reporting biases. For several countries with high numbers of introduced ungulate species, such as South Africa, France, Germany, the Russian Federation, Cuba, Papua New Guinea and Columbia, we found no, or few, impact reports (Fig. 3). We found a continental reporting bias, where Asia, Africa and Europe had fewer impact reports than other continents relative to their number of introduced ungulate species, while Northern America was the most-studied continent (Fig. S1 in Supp. 1). Local extirpations of native populations (Major impacts) have been reported in Europe (6: Ireland, United Kingdom and Czech Republic), Northern America (5: United States and Canada), Oceania (2: Australia and New Caledonia) and South America (1: Brazil). Of all the species that caused local population extirpations, only sika deer caused them in different regions (in 4 out of the 23 regions with impact observations; Table 2).

The impact of widely introduced species (i.e. species introduced to many countries) was generally more often studied than the impact of species introduced to few countries ( $p<001$; Table S12 in Supp. 1). By contrast, species having caused local extinctions were not more often studied than other species ( $p=0.14$, Table S13 in Supp. 1$)$, as more widely introduced species did not cause more local extinctions ( $p=0.12$, Table S14 in Supp. 1 ).

Comparisons between alien species within impact categories: Incorporating risk in comparisons between species

Classifying and comparing ungulates based on their highest impact magnitude, as proposed in the current EICAT procedure, resulted in only three categories (Table 2): only one species (4\%) did not cause a higher impact than a decreased performance of native individuals (classified in the Minor category), two thirds of the species (18 species; $66 \%$ ) caused at least once a native population decline (Moderate category), and about one third (8 species; $30 \%$ ) caused at least once a (reversible) local extinction (Major category).

When further ranking ungulates within each of these three categories based on their impact risks, i.e. how frequently they would cause their highest impact magnitude, the mouflon was ranked highest among the seven species of the 
Major category, with the dromedary ranking second (Table 2). Due to the generally low numbers of regions with impact observations for each species, a high uncertainty existed regarding their risks of causing Major impacts. Species with impact observations from less than five regions, such as the mouflon, dromedary, Javan deer and white-tailed deer, had the widest $95 \% \mathrm{Cls}$ (Fig. 4a), resulting in very little information about their risk of causing Major impacts. The narrower $\mathrm{Cls}$ of cattle and sheep (impact observations from 5 to 10 regions; Fig. 4b) indicated a slightly higher confidence about their impact risks, and the relatively narrow $\mathrm{Cls}$ of the goat and sika deer (impact observations from $>10$ regions) provided the most informative impact risks in the Major category (i.e. impact risks with lowest uncertainty) (Fig. 4c). Species' pairwise comparisons revealed that the mouflon had a significantly higher risk of causing Major impacts than the goat and sika deer, and that the dromedary had a significantly higher risk than the goat (Table S15 in Supp. 1). The impact risks of the other species classified in the Major category did not significantly differ from each other (Table S15 in Supp. 1).

Within the Moderate category, most species (12/18) had few regions with impact observations (<5), providing little information about their risk to cause Moderate impacts (Fig. 4d-f). Therefore, pairwise comparisons between the species classified in the Moderate category revealed that their impact risks were rarely significantly different (Table S16). The only significant differences concerned the donkey (Equus asinus, Linnaeus, 1758), ranked as the least detrimental species of the Moderate category, which was found to have a lower impact risk than the reindeer (Rangifer tarandus, Linnaeus, 1758), water buffalo (Bubalus bubalis, Linnaeus, 1758), chital (Axis axis, Erxleben, 1777), fallow deer (Dama dama, Linnaeus, 1758) and red deer (Cervus elaphus, Linnaeus 1758) (Table S16 in Supp. 1). The wild boar had the most numerous impact observations (63 impact observations) and still never reached a higher impact than the Moderate category.

\section{Discussion}

We used the EICAT classification to compare 441 impacts caused by 27 alien ungulates on the native fauna and flora in their recipient environments, by systematically classifying them into five impact magnitudes. Based on these individual impacts, an overall environmental impact should be assigned to each alien species, to enable comparisons between them and the prioritization of the most damaging ones. EICAT only discriminates five impact categories, by comparing species based on their highest impact magnitude (IUCN, 2020b), which limits its practical use for prioritization if many species are compared. When comparing alien ungulates based on their highest impact magnitude, 26 out of 27 species were classified in the harmful EICAT categories (i.e. MO, MR and MV). Comparisons of impacts across taxa (e.g. an animal species vs a plant species) are crucial for informing decisions about which species to manage, as resources for management are usually allocated to priority alien species without taxonomic distinction (Kumschick et al., 2015). However, if too many species end up in high-impact categories (which is likely once many species from different taxa will be assessed), EICAT classifications become uninformative for prioritization. When we compare the EICAT impacts of alien ungulate species with the EICAT impacts of species from other taxonomic groups for which global assessments exist, such as alien amphibians (Kumschick et al., 2017), birds (Evans et al., 2016) and bamboos (Canavan, Kumschick, Le Roux, et al., 2019), a total of 196 species are classified within the five EICAT categories (Table 3): almost half (44\%) of the species are classified in the harmful categories.

\section{Improvement of the EICAT ranking: impact risks}

Integrating impact risk allowed the consideration of the variation in species' impacts, which is ignored in final EICAT scores. Based on this risk, we discriminated among species that have caused local extirpations of native species, which are all scored in the same EICAT category. The mouflon was identified as the worst alien ungulate. We showed that the mouflon is more likely than the goat and sika deer to cause native local extirpations, and that the donkey is less likely to cause native population declines than the reindeer, water buffalo, chital, fallow deer and red deer. Similarly, we found that it is unlikely for the wild boar to cause Major impacts; although it is the species with the most impact observations, none of them documented a Major impact. However, as most ungulate species were only studied in few 
regions (63\% had impact observations from less than five regions), we did not find other significant differences between their impact risks, because of the large overlap of their wide Cls.

EICAT identifies knowledge gaps for species with no impact reports as they are classified as Data Deficient; for the IUCN Red List, the classification of species within the Data Deficient category has been shown to efficiently redirect priorities of research efforts towards these species (Jarić et al., 2017). Considering the impact risk allowed to identify knowledge gaps for species with impact reports only available for a small region of their total introduced range. Some species have rarely been introduced (e.g. Asian elephant (Elephas maximus, Linnaeus, 1758) or gemsbok (Oryx gazella, Linnaeus, 1758); Table S3 in Supp. 1); however, other widely introduced species, such as the mouflon, white-tailed deer or water buffalo (Table S3 in Supp. 1), have been studied in only few of the regions to which they were introduced. This prevents the accurate evaluation of their impact risks, because information on the variation in their impacts is not available. The impact of such species needs to be described in other parts of their introduced range to get more representative data on their impacts and for improved comparisons with other species. The number of data points largely determines the width of the Cls, thus, adding more impact reports from a variety of regions to the database will be the most efficient way to improve impact rankings. Like the EICAT classification (Hawkins et al., 2015), our assessment of the impact risks of alien ungulates should be dynamic and updated with new observations, and the ranking should be adapted based on new evidence.

The ranking of the worst ungulates presented in this article is only based on the species' risks of causing impacts. However, other aspects must be considered in management decisions, such as the management feasibility and costs, or the species' risk of being introduced and of establishing and spread. Several frameworks for have been developed to combine all these different aspects in the decision process (e.g. Kumschick et al., 2020; Bertolino et al., 2020).

\section{Comparisons with other rankings}

Our ranking of alien ungulates with the highest impacts can be compared with other rankings. Several non-quantitative listings of the most detrimental aliens have been produced based on expert opinion: the IUCN Invasive Species Specialist Group (ISSG) listed the goat, red deer and wild boar on their '100 of the world's worst invasive alien species' list (Lowe et al., 2000; Luque et al., 2014). At the European scale, Carboneras et al. (2018) established a prioritization list for risk assessments, with the sika deer and American bison evaluated as first priority species, and the chital and dromedary as second priority species, because of their high impacts. While these listings are consistent with our ranking regarding some species (e.g. sika deer, dromedary and goats as priority species), the selection of other species might have been subject to expert opinions rather than based on evidence of high impacts (Nentwig et al., 2010). For instance, the species ubiquity might have biased expert opinions, as the wild boar, red deer and chital are all part of the 12 most widely introduced ungulates (Table S3 in Supp. 1).

Bellard, Genovesi and Jeschke (2016) evaluated the most damaging aliens to vertebrates by analysing their main threats listed in the Global Invasive Species Database (GISD) and IUCN Red List. They evaluated wild boar, goat and cattle as the fifth, sixth and respectively seventh most threatening alien species (i.e. threatening the largest numbers of vertebrate species; after the amphibian chytrid fungus (Batrachochytrium dendrobatidis), rats, cats and dogs). In our study, we found the sika deer to have caused several extinctions of red deer populations through hybridization, making it the most damaging alien ungulate for native vertebrates based on our criteria. We found wild boars to affect vertebrates in 23 impact observations, goats in 11 observations and cattle in only one: neither species caused local extinctions; the highest impacts were declines in vertebrate populations. Still, consistent with the findings of Bellard et al. (2016), we also found wild boars and goats to represent important threats to native vertebrates on islands: they both led to population declines of vertebrates on almost half of the archipelagos they have been introduced to (6/15 archipelagos compared to $1 / 5$ mainland regions for goats; $4 / 12$ archipelagos compared to $0 / 11$ mainland regions for wild boars).

Nentwig et al. (2018) ranked 498 invasive alien species introduced to Europe, from the most to the least detrimental, based on their environmental and socio-economic impacts, classified with the Generic Impact Scoring System (GISS; Nentwig et al., 2010; Nentwig et al., 2016). GISS is an additive scoring system which classifies impacts from 0 to 5 via 
12 mechanisms: the overall impact of an alien is summarized by summing the highest score of each mechanism. Nentwig et al. (2018) ranked species by combining two ranking approaches: based on their GISS overall scores and by the number of the highest impact scores (i.e. maximum impact, analogous to the EICAT procedure). Six ungulates were listed among the 149 worst aliens (excluding domestic species and only considering species alien to - and successfully introduced in - Europe). In order of the most to the least detrimental, these were: sika deer, reeves' muntjac (Muntiacus reevesi, Ogilby, 1839), chital, aoudad (Ammotragus lervia, Pallas, 1777), white-tailed deer and mouflon. Our ranking of these species is quite different (Table 2). As highlighted by Nentwig et al. (2018), the two ranking approaches they used have benefits: GISS overall scores inform on the variation in the species impact, whereas maximum impacts identify species with potential of causing high impacts. However, the drawbacks of additive scoring systems in prioritization are well known (e.g. Game et al., 2013). The GISS overall score is indeed biased: widely studied species will rank higher than poorly studied species (as mechanisms without data score 0 ) and species causing impacts through multiple mechanisms will rank higher than species causing impacts through a single mechanism. Our ranking system captures intraspecific variation without adopting an additive system and captures uncertainty associated with small sampling effort.

\section{Reporting biases}

\section{Indirect impacts are reported mainly when they are severe}

Native animals were found to be more severely impacted by alien ungulates than native plants, because, in contrast to native plants, they were mainly affected via indirect mechanisms. An explanation for indirect impacts to be associated with more severe impacts might be that indirect negligible or minor impacts are difficult to detect. When interactions between species can be directly observed (e.g. an alien deer grazing on a native plant), impacts are assumed and studied. However, for indirect impacts to be studied they must first be detected, which is easier if impacts are severe. Moreover, as indirect impacts are more difficult to demonstrate, studies usually focus on studying the mechanisms, rather than on quantifying impact magnitudes. Spear and Chown (2009) also observed a lack of robust evidence for impacts occurring through competition on the native fauna in contrast to direct impacts to the native vegetation through herbivory.

\section{Biases in impact magnitudes}

A potential bias towards studies requiring simpler designs has already been highlighted by Measey et al. (2020), who found that studies showing native local extinctions are more costly because of the complexity of their designs. In our study, we indeed found only few observations of native local extinctions in comparison to other impact magnitudes. This alone does not indicate a bias, as it is likely that local extinctions caused by alien species occur less frequently than lower impacts. However, for $19 \%$ of the observations classified with a Moderate impact magnitude, the assessor specified that the impact might have been higher (i.e. that the alien might have led to a local extinction), but that the design of the study did not allow to determine whether this was the case (see EICAT assessments, Sheet 4 in Supp. 2). Similarly, for $91 \%$ of the Minor impacts, the assessor specified that the impact might have been higher but that the study did not investigate changes at the native population level (or not adequately). Thus, native local extinctions and population declines might have been under-evaluated, probably because most impact reports focus on what is easier and less costly to demonstrate (Measey et al., 2020).

On the other hand, it is likely that negligible impacts (Minimal Concern impacts) are not frequently reported as studies will focus on demonstrating impacts rather than the absence of impact, i.e. the so-called file drawer problem (Sterling, 1959). In our study, only $7 \%$ of impact observations reported negligible impacts, which might be a strong underrepresentation. This is also seen in the IUCN Red List where research effort is biased towards species expected to be threatened (Bachman et al., 2019). To prevent this bias, the IUCN Red List recently reduced the data requirements for assessing non-threatened species ('Least Concern' category) (IUCN, 2016; Bachman et al., 2019), and approaches to rapidly assess these species from open-sources databases have been developed (Rivers 2017; Bachman et al., 2020). Such rapid assessment approaches for negligible impacts of alien populations could be developed in EICAT, in order to decrease this bias and improve the quantity of available information about the intraspecific variation in impacts. 
Data availability concerning impacts of aliens has been shown to be unevenly distributed around the world (Pyšek et al., 2008, Hulme et al., 2013) and in alien birds, mainly determined by their alien range (Evans, Pigot, Kumschick, Şekercioğlu, \& Blackburn, 2018; Evans \& Blackburn, 2020). Like for other taxa (Pyšek et al., 2008; Evans \& Blackburn, 2020), we found that impacts were more frequently studied in regions with more introduced ungulate species. We also found that Asia and Africa were understudied compared to other continents: this is often explained by the generally lower economic development and wealth of these continents resulting in a smaller research effort (Pyšek et al., 2008; Evans \& Blackburn, 2020). However, we also found impacts of alien ungulates to be relatively less studied in Europe. This might indicate a language bias, where reports in their regional language might not often be translated into English and thereby poorly represented in the literature. This is supported by the fact that the most-studied countries were all English-speaking (United States, New Zealand, United Kingdom, Australia and Canada; Fig. 3). Increased efforts should be made to identify and include non-English reports in assessments to counter this language bias.

Like for alien birds (Evans, Pigot, et al., 2018), we found the impact of widely-introduced alien species to generally be more studied than the impact of rarely-introduced species. This might be also true when comparing different taxonomic groups: alien ungulates are among the most widely introduced groups and we found them to be equally well, or better, studied than alien birds, amphibians and bamboos: $59 \%$ of the ungulate species were classified as Data Deficient, compared to $62 \%$ for amphibians (Kumschick et al., 2017), $71 \%$ for birds (Evans et al., 2016) and $85 \%$ for bamboos (Canavan, Kumschick, Roux, et al., 2019). This does not necessarily indicate a bias, as more widely introduced species or taxa likely cause more numerous impacts overall, because of their multiple introduced populations. Also, the more widely a species is introduced, the higher is its chance to be introduced to a country where impacts of aliens are well-studied. By contrast, unlike previous findings on birds (Evans, Pigot et al., 2018) and plants (Pyšek et al., 2008), we did not find alien ungulates causing higher impacts to be more studied.

\section{Study limitations}

So far, the EICAT system does not propose a method to consider different levels of uncertainty associated with impact assessments in the final species ranking. Hence, all impact reports were given the same weight when summarizing the results, regardless of the confidence assigned to them. Half of the impact observations were assigned a low confidence score, and 5 out of the 8 species of the Major category (Javan deer, mouflon, cattle, sheep, goat) were classified based on impact observations with low confidence scores. However, we did not find differences in the distribution of confidence levels across impact magnitudes, indicating no general bias in our species ranking due to uncertainty associated with the quality of the EICAT assessments. Ultimately, this type of uncertainty would need to be considered in the final ranking, as it might make sense to prioritize species causing high impacts with high certainty over species with uncertain impacts (Probert et al., 2020).

\section{Future research: towards predictions of impacts}

\section{Comparing impacts of different taxonomic groups}

Impact magnitudes are not randomly distributed among taxa. Comparing our results to other vertebrates that were also assessed using EICAT, alien ungulates caused higher impacts than alien birds and amphibians $(p<0.001$; Table S17 in Supp. 1). These findings corroborate previous studies at the European level that alien mammals generally cause higher impacts than other animals (Kumschick et al., 2015). Even though great variation among species has been found within all the compared taxonomic groups (see Table 3), comparisons between taxonomic groups (mammals vs birds and amphibians) can help improving our general understanding of impacts. For instance, the generally higher impacts of alien ungulates could be explained by their role as ecosystem engineers and their large influences on community compositions and ecosystem processes (e.g. nitrogen cycle or fire regime), through selective foraging, seed dispersal, trampling, etc. (e.g. Velamazán et al., 2020; Lecomte et al., 2019; Rooney, 2009). 


\section{Understanding impacts by studying context}

In this study, we aimed at approximating the global impact risks of alien ungulates. When setting priorities for regional management, it is important to compare species only based on impacts potentially relevant for the region of interest. To achieve this, we need to understand the conditions associated with high and low impacts. EICAT (and GISS) assessments have already been used in attempts to explain variation in impact magnitude across species, but not within species (EICAT: Evans et al., 2016; Evans, Kumschick, et al., 2018; Kesner \& Kumschick, 2018; Kumschick et al., 2017; GISS: Nentwig et al. 2010; Kumschick et al., 2015; Nentwig et al., 2018). Ignoring intraspecific variation represents a loss of crucial information when context-dependency is investigated. In this study, we provide a dataset capturing inter- and intraspecific variation in impacts. Future research can build on this dataset to identify potential drivers of this variation, across and within alien ungulate species. A recent framework identified three types of factors and their interactions that explain invasions: alien species traits, location characteristics, and event-related factors (Pyšek et al., 2020). This framework can guide the quest for a better understanding of the context-dependency of impact magnitudes.

\section{Global Impact Database}

Many EICAT studies provided little information on the assessments and did not publish their raw data; most of them only provided the highest impact magnitude per species (Evans et al., 2016; Kesner \& Kumschick, 2018; Kumschick et al., 2017; Nelufule et al., 2020; but see Canavan et al., 2019; Galanidi et al., 2018; Hagen \& Kumschick, 2018). This prevents others from calculating the impact risk of species for cross-taxonomic comparisons or study context dependence of impacts. Likewise, some studies did not provide uncertainty estimates (Hagen \& Kumschick, 2018; Kumschick et al., 2017; Nelufule et al., 2020), preventing quality checks of classifications and leaving room for inconsistencies (González-Moreno et al., 2019). Thus, incomplete publication of raw data from EICAT assessments limits the progress in understanding the variation of impacts. We strongly recommend that future studies performing EICAT assessments provide complete assessments, encompassing all impact observations - and not only the highest to have a full picture of the impacts of an alien species and to allow relevant analyses of these.

We advocate the creation of a public, freely-accessible Global Impact Database in which all impact reports could be deposited (see also Strubbe et al., 2019). EICAT could play a pivotal role in that it offers a standardized way of collecting data. A Global Impact Database will render the information directly accessible to policy makers and the public (instead of distributed in scientific publications; Cadotte et al., 2020) and will allow global analyses looking for potential patterns in impacts across alien taxa and/or recipient environments.

\section{Authors' contribution}

LV and SBa conceived the study; LV, DJ, LS collected data; LV and SBa analysed data; LV, SBe, SBa wrote the first draft; all authors commented and approved the final paper.

\section{Acknowledgments}

The authors thank Rudolph Rohr for his statistical support and Anna Frances Probert for her review of the manuscript. The authors also thank Shyama Pagad and Lucilla Carnevali for providing us ungulate data from the GRIIS database, and Alix Badel and Maddie Smith for their help with literature search and EICAT assessments. LV and SB acknowledge funding from the Swiss National Science Foundation (grant numbers 31003A_179491 and 31BD30_184114) and the Belmont Forum - BiodivERsA International joint call project InvasiBES (PCI2018-092939). LS acknowledges funding from the European Cooperation in Science and Technology (COST) Action TD1209 (ECOST-STSM-TD1209-030417-084509). 


\section{References}

Bachman, S. P., Field, R., Reader, T., Raimondo, D., Donaldson, J., Schatz, G. E., \& Lughadha, E. N. (2019). Progress, challenges and opportunities for Red Listing. Biological Conservation, 234, 45-55. doi: 10.1016/j.biocon.2019.03.002

Bachman, S., Walker, B. E., Barrios, S., Copeland, A., \& Moat, J. (2020). Rapid Least Concern: towards automating Red List assessments. Biodiversity Data Journal, 8. doi: 10.3897/BDJ.8.e47018

Bates, D., Mächler, M., Bolker, B., \& Walker, S. (2014). Fitting Linear Mixed-Effects Models using Ime4. ArXiv:1406.5823 [Stat]. http://arxiv.org/abs/1406.5823

Bellard, C., Cassey, P., \& Blackburn, T. M. (2016). Alien species as a driver of recent extinctions. Biology Letters, 12(2), 20150623. doi: 10.1098/rsbl.2015.0623

Bellard, C., Genovesi, P., \& Jeschke, J. M. (2016). Global patterns in threats to vertebrates by biological invasions. Proceedings of the Royal Society B: Biological Sciences, 283(1823), 20152454. doi: 10.1098/rspb.2015.2454

Bertolino S., Ancillotto L., Bartolommei P., Benassi G., Capizzi D., Gasperini S., Lucchesi M., Mori E., Scillitani L., Sozio G., Falaschi M., Ficetola G.F., Cerri J., Genovesi P., Carnevali L., Loy A., Monaco A. 2020. A framework for prioritising present and potentially invasive mammal species for a national list. NeoBiota 62: 31-54. doi: 10.3897/neobiota.62.52934

Blackburn, T. M., Essl, F., Evans, T., Hulme, P. E., Jeschke, J. M., Kühn, I., Kumschick, S., Marková, Z., Mrugała, A., Nentwig, W., Pergl, J., Pyšek, P., Rabitsch, W., Ricciardi, A., Richardson, D. M., Sendek, A., Vilà, M., Wilson, J. R. U., Winter, M., ... Bacher, S. (2014). A Unified Classification of Alien Species Based on the Magnitude of their Environmental Impacts. PLoS Biology, 12(5), e1001850. doi: 10.1371/journal.pbio.1001850

Cadotte, M. W., Jones, H. P., \& Newton, E. L. (2020). Making the applied research that practitioners need and want accessible. Ecological Solutions and Evidence, 1(1), e12000. doi: 10.1002/2688-8319.12000

Canavan, S., Kumschick, S., Roux, J. J. L., Richardson, D. M., \& Wilson, J. R. U. (2019). Does origin determine environmental impacts? Not for bamboos. Plants, People, Planet, 1(2), 119-128. doi: 10.1002/ppp3.5

Carboneras, C., Genovesi, P., Vilà, M., Blackburn, T. M., Carrete, M., Clavero, M., D’hondt, B., Orueta, J. F., Gallardo, B., Geraldes, P., González-Moreno, P., Gregory, R. D., Nentwig, W., Paquet, J.-Y., Pyšek, P., Rabitsch, W., Ramírez, I., Scalera, R., Tella, J. L., ... Wynde, R. (2018). A prioritised list of invasive alien species to assist the effective implementation of EU legislation. Journal of Applied Ecology, 55(2), 539-547. doi: 10.1111/1365-2664.12997

Davis, N. E., Forsyth, D. M., \& Coulson, G. (2010). Facilitative interactions between an exotic mammal and native and exotic plants: Hog deer (Axis porcinus) as seed dispersers in south-eastern Australia. Biological Invasions, 12(5), 1079-1092. doi: 10.1007/s10530-009-9525-1

Evans, T., \& Blackburn, T. M. (2020). Global variation in the availability of data on the environmental impacts of alien birds. Biological Invasions, 22(3), 1027-1036. doi: 10.1007/s10530-019-02153-z

Evans, T., Kumschick, S., \& Blackburn, T. M. (2016). Application of the Environmental Impact Classification for Alien Taxa (EICAT) to a global assessment of alien bird impacts. Diversity and Distributions, 22(9), 919-931. doi: 10.1111/ddi.12464

Evans, T., Kumschick, S., Şekercioğlu, Ç. H., \& Blackburn, T. M. (2018). Identifying the factors that determine the severity and type of alien bird impacts. Diversity and Distributions, 24(6), 800-810. doi: 10.1111/ddi.12721

Evans, T., Pigot, A., Kumschick, S., Şekercioğlu, Ç. H., \& Blackburn, T. M. (2018). Determinants of data deficiency in the impacts of alien bird species. Ecography, 41(8), 1401-1410. doi: 10.1111/ecog.03232

Galanidi, M., Zenetos, A., \& Bacher, S. (2018). Assessing the socio-economic impacts of priority marine invasive fishes in the Mediterranean with the newly proposed SEICAT methodology. Mediterranean Marine Science, 19(1), 107. doi: $10.12681 / \mathrm{mms} .15940$

Game, E. T., Kareiva, P., \& Possingham, H. P. (2013). Six Common Mistakes in Conservation Priority Setting. Conservation Biology, 27(3), 480-485. doi: 10.1111/cobi.12051

González-Moreno, P., Lazzaro, L., Vilà, M., Preda, C., Adriaens, T., Bacher, S., Brundu, G., Copp, G. H., Essl, F., GarcíaBerthou, E., Katsanevakis, S., Moen, T. L., Lucy, F. E., Nentwig, W., Roy, H. E., Srèbalienè, G., Talg $\varnothing$, V., Vanderhoeven, S., Andjelković, A., ... Kenis, M. (2019). Consistency of impact assessment protocols for nonnative species. NeoBiota, 44, 1-25. doi: 10.3897/neobiota.44.31650

Hagen, B. L., \& Kumschick, S. (2018). The relevance of using various scoring schemes revealed by an impact assessment of feral mammals. NeoBiota, 38, 35-75. doi: 10.3897/neobiota.38.23509 
Hartig, F. (2020). DHARMa: Residual Diagnostics for Hierarchical (Multi-Level / Mixed) Regression Models. R package version 0.3.3.0. https://CRAN.R-project.org/package=DHARMa

Hawkins, C. L., Bacher, S., Essl, F., Hulme, P. E., Jeschke, J. M., Kühn, I., Kumschick, S., Nentwig, W., Pergl, J., Pyšek, P., Rabitsch, W., Richardson, D. M., Vilà, M., Wilson, J. R. U., Genovesi, P., \& Blackburn, T. M. (2015). Framework and guidelines for implementing the proposed IUCN Environmental Impact Classification for Alien Taxa (EICAT). Diversity and Distributions, 21(11), 1360-1363. doi: 10.1111/ddi.12379

Hemming, V., Burgman, M. A., Hanea, A. M., McBride, M. F., \& Wintle, B. C. (2018). A practical guide to structured expert elicitation using the IDEA protocol. Methods in Ecology and Evolution, 9(1), 169-180. doi: 10.1111/2041210X.12857

Hulme, P. E., Pyšek, P., Jarošík, V., Pergl, J., Schaffner, U., \& Vilà, M. (2013). Bias and error in understanding plant invasion impacts. Trends in Ecology \& Evolution, 28(4), 212-218. doi: 10.1016/j.tree.2012.10.010

IUCN. (2016). Rules of Procedure for IUCN Red List Assessments 2017-2020. Version 3.0. Approved by the IUCN SSC Steering Committee in September 2016.

IUCN. (2020a). Guidelines for using the IUCN Environmental Impact Classification for Alien Taxa (EICAT) Categories and Criteria): First edition. Gland, Switzerland and Cambridge, UK: IUCN.

IUCN. (2020b). IUCN EICAT Categories and Criteria. The Environmental Impact Classification for Alien Taxa (EICAT): First edition. Gland, Switzerland and Cambridge, UK: IUCN.

Jarić, I., Roberts, D. L., Gessner, J., Solow, A. R., \& Courchamp, F. (2017). Science responses to IUCN red listing. PeerJ, 5, e4025. doi: 10.7717/peerj.4025

Kesner, D., \& Kumschick, S. (2018). Gastropods alien to South Africa cause severe environmental harm in their global alien ranges across habitats. Ecology and Evolution, 8(16), 8273-8285. doi: 10.1002/ece3.4385

Kulhanek, S. A., Ricciardi, A., \& Leung, B. (2011). Is invasion history a useful tool for predicting the impacts of the world's worst aquatic invasive species? Ecological Applications, 21(1), 189-202. doi: 10.1890/09-1452.1

Kumschick, S., Bacher, S., Evans, T., Marková, Z., Pergl, J., Pyšek, P., Vaes-Petignat, S., Veer, G. van der, Vilà, M., \& Nentwig, W. (2015). Comparing impacts of alien plants and animals in Europe using a standard scoring system. Journal of Applied Ecology, 52(3), 552-561. doi: 10.1111/1365-2664.12427

Kumschick, S., Vimercati, G., de Villiers, F. A., Mokhatla, M. M., Davies, S. J., Thorp, C. J., Rebelo, A. D., \& Measey, G. J. (2017). Impact assessment with different scoring tools: How well do alien amphibian assessments match? NeoBiota, 33, 53-66. doi: 10.3897/neobiota.33.10376

Kumschick, S., Wilson, J. R., \& Foxcroft, L. C. (2020). A framework to support alien species regulation: the Risk Analysis for Alien Taxa (RAAT). NeoBiota, 62, 213. doi: 10.3897/neobiota.62.51031

Lecomte, X., Caldeira, M. C., Catry, F. X., Fernandes, P. M., Jackson, R. B., \& Bugalho, M. N. (2019). Ungulates mediate trade-offs between carbon storage and wildfire hazard in Mediterranean oak woodlands. Journal of Applied Ecology, 56(3), 699-710. doi: 10.1111/1365-2664.13310

Long, J. L. (2003). Introduced mammals of the world: Their history distribution and influence. CSIRO PUBLISHING and CABI Publishing.

Lowe, S., Browne, M., Boudjelas, S., \& De Poorter, M. (2000). 100 of the world's worst invasive alien species: A selection from the global invasive species database. Published by The Invasive Species Specialist Group (ISSG) a specialist group of the Species Survival Commission (SSC) of the World Conservation Union (IUCN), 12pp. First published as special lift-out In Aliens 12, December 2000. Updated and reprinted version: November 2004. Journal of Experimental Marine Biology and Ecology 258, 3954.

Loydi, A., \& Zalba, S. M. (2009). Feral horses dung piles as potential invasion windows for alien plant species in natural grasslands. Plant Ecology, 201(2), 471-480. doi: 10.1007/s11258-008-9468-0

Luque, G. M., Bellard, C., Bertelsmeier, C., Bonnaud, E., Genovesi, P., Simberloff, D., \& Courchamp, F. (2014). The 100th of the world's worst invasive alien species. Biological Invasions, 16(5), 981-985. doi: 10.1007/s10530-0130561-5

Measey, J., Wagener, C., Mohanty, N. P., Baxter-Gilbert, J., \& Pienaar, E. F. (2020). The cost and complexity of assessing impact. NeoBiota, 62, 27. doi: 10.3897/neobiota.62.52261

Nelufule, T., Robertson, M. P., Wilson, J. R. U., Faulkner, K. T., Sole, C., \& Kumschick, S. (2020). The threats posed by the pet trade in alien terrestrial invertebrates in South Africa. Journal for Nature Conservation, 55, 125831. doi: 10.1016/j.jnc.2020.125831 
Nelufule, T., Robertson, M. P., Wilson, J. R., \& Kumschick, S. (2018). An assessment of alien terrestrial invertebrate species in the pet trade in South Africa [Master of Science in Zoology]. University of Pretoria.

Nentwig, W., Bacher, S., Kumschick, S., Pyšek, P., \& Vilà, M. (2018). More than "100 worst" alien species in Europe. Biological Invasions, 20(6), 1611-1621. doi: 10.1007/s10530-017-1651-6

Nentwig, W., Bacher, S., Pyšek, P., Vilà, M., \& Kumschick, S. (2016). The generic impact scoring system (GISS): A standardized tool to quantify the impacts of alien species. Environmental Monitoring and Assessment, 188(5), 315. doi: 10.1007/s10661-016-5321-4

Nentwig, W., Kühnel, E., \& Bacher, S. (2010). A Generic Impact-Scoring System Applied to Alien Mammals in Europe. Conservation Biology, 24(1), 302-311. doi: 10.1111/j.1523-1739.2009.01289.x

O'Connor, S.-J., \& Kelly, D. (2012). Seed dispersal of matai (Prumnopitys taxifolia) by feral pigs (Sus scrofa). New Zealand Journal of Ecology, 36(2), 228-231.

Parker, I. M., Simberloff, D., Lonsdale, W. M., Goodell, K., Wonham, M., Kareiva, P. M., Williamson, M. H., Holle, B. V., Moyle, P. B., Byers, J. E., \& Goldwasser, L. (1999). Impact: Toward a Framework for Understanding the Ecological Effects of Invaders. Biological Invasions, 1(1), 3-19.

Probert, A. F., Volery, L., Kumschick, S., Vimercati, G., \& Bacher, S. (2020). Understanding uncertainty in the Impact Classification for Alien Taxa (ICAT) assessments. NeoBiota, 62, 387. doi: 10.3897/neobiota.62.52010

Pyšek, P., Bacher, S., Kühn, I., Novoa, A., Catford, J. A., Hulme, P. E., Pergl, J., Richardson, D. M., Wilson, J. R. U., \& Blackburn, T. M. (2020). MAcroecological Framework for Invasive Aliens (MAFIA): Disentangling large-scale context dependence in biological invasions. NeoBiota, 62, 407. doi: 10.3897/neobiota.62.52787

Pyšek, P., Richardson, D. M., Pergl, J., Jarošík, V., Sixtová, Z., \& Weber, E. (2008). Geographical and taxonomic biases in invasion ecology. Trends in Ecology \& Evolution, 23(5), 237-244. doi: 10.1016/j.tree.2008.02.002

$\mathrm{R}$ Core Team. (2019). R: A language and environment for statistical computing. R Foundation for Statistical Computing. https://www.R-project.org/

Ricciardi, A. (2003). Predicting the impacts of an introduced species from its invasion history: An empirical approach applied to zebra mussel invasions. Freshwater Biology, 48(6), 972-981. doi: 10.1046/j.13652427.2003.01071.x

Ricciardi, A., Hoopes, M. F., Marchetti, M. P., \& Lockwood, J. L. (2013). Progress toward understanding the ecological impacts of nonnative species. Ecological Monographs, 83(3), 263-282. doi: 10.1890/13-0183.1

Rivers, M. (2017). The Global Tree Assessment-red listing the world's trees. BGJournal, 14(1), 16-19.

Rooney, T. P. (2009). High white-tailed deer densities benefit graminoids and contribute to biotic homogenization of forest ground-layer vegetation. Plant Ecology, 202(1), 103-111. doi: 10.1007/s11258-008-9489-8

Roy, H. E., European Commission, Directorate-General for the Environment, \& CEH (Centre of Ecology \& Hydrology). (2015). Invasive alien species: Prioritising prevention efforts through horizon scanning: final report. Publications Office. http://dx.publications.europa.eu/0.2779/096586

Roy, H. E., Peyton, J., Aldridge, D. C., Bantock, T., Blackburn, T. M., Britton, R., Clark, P., Cook, E., Dehnen-Schmutz, K., Dines, T., Dobson, M., Edwards, F., Harrower, C., Harvey, M. C., Minchin, D., Noble, D. G., Parrott, D., Pocock, M. J. O., Preston, C. D., ... Walker, K. J. (2014). Horizon scanning for invasive alien species with the potential to threaten biodiversity in Great Britain. Global Change Biology, 20(12), 3859-3871. doi: 10.1111/gcb.12603

Seebens, H., Blackburn, T. M., Dyer, E. E., Genovesi, P., Hulme, P. E., Jeschke, J. M., Pagad, S., Pyšek, P., Winter, M., Arianoutsou, M., Bacher, S., Blasius, B., Brundu, G., Capinha, C., Celesti-Grapow, L., Dawson, W., Dullinger, S., Fuentes, N., Jäger, H., ... Essl, F. (2017). No saturation in the accumulation of alien species worldwide. Nature Communications, 8(1), 1-9. doi: 10.1038/ncomms14435

Spear, D., \& Chown, S. L. (2009). Non-indigenous ungulates as a threat to biodiversity. Journal of Zoology, 279(1), 117. doi: 10.1111/j.1469-7998.2009.00604.x

Sterling, T. D. (1959). Publication decisions and their possible effects on inferences drawn from tests of significanceor vice versa. Journal of the American statistical association, 54(285), 30-34.

Strubbe, D., White, R., Edelaar, P., Rahbek, C., \& Shwartz, A. (2019). Advancing impact assessments of non-native species: Strategies for strengthening the evidence-base. NeoBiota, 51, 41-64. doi: 10.3897/neobiota.51.35940

Sundar, D. (2014). binom: Binomial Confidence Intervals For Several ParameterizationsR package version 1.1-1. https://CRAN.R-project.org/package=binom 
Velamazán, M., Perea, R., \& Bugalho, M. N. (2020). Ungulates and ecosystem services in Mediterranean woody systems: A semi-quantitative review. Journal for Nature Conservation, 125837. doi: 10.1016/j.jnc.2020.125837

Volery, L., Blackburn, T. M., Bertolino, S., Evans, T., Genovesi, P., Kumschick, S., Roy, H. E., Smith, K. G., \& Bacher, S. (2020). Improving the Environmental Impact Classification for Alien Taxa (EICAT): A summary of revisions to the framework and guidelines. NeoBiota, 62, 547. doi: 10.3897/neobiota.62.52723

Warren, R. J., King, J. R., Tarsa, C., Haas, B., \& Henderson, J. (2017). A systematic review of context bias in invasion biology. PLoS One, 12(8), e0182502. doi: 10.1371/journal.pone.0182502

Zablotskaya, L. V., Zablotsky, M. A., \& Zablotskaya, M. M. (2004). Origin of the Hybrids of North American and European Bison in the Caucasus Mountains. In European Bison. Status Survey and Conservation Action Plan. (Pucek, Z., pp. 49-52). IUCN.

Zhong, H., \& Song, M. (2019). A Fast Exact Functional Test for Directional Association and Cancer Biology Applications. IEEE/ACM Transactions on Computational Biology and Bioinformatics, 16(3), 818-826. doi: 10.1109/TCBB.2018.2809743 
Table 1. The five steps impact magnitude classification of EICAT: the categories MO, MR and MV are considered 'harmful' categories (adapted from IUCN 2020a).

\section{Impact magnitude Meaning/Criteria}

The alien causes negligible levels of impacts, but does not affect the individual

Minimal Concern (MC) performance of natives (i.e. their capacity to survive, gather resources, grow, or reproduce).

\section{Minor (MN)}

Moderate (MO)

Major (MR)
The alien causes reductions in the performance of native individuals, but does not cause any decline in any native population.

The alien causes a decline in at least one native population, but no local extinction of any population.

The alien causes a local extinction of at least one native population; this local extinction is reversible (i.e. if the alien population was no longer present in the area, the native population would be likely to recolonize the area through natural dispersal processes within three generations or 10 years, whichever is longer).

The alien causes an irreversible local extinction of at least one native population (i.e. if the alien population was no longer present in the area, the native population would not be likely to recolonize the area, for instance because of little demographic exchange between sub-populations). 
Table 2. Results of the EICAT classification of the 27 assessed ungulate species, and the ranking of species from the most to the least detrimental. Species were first ranked into three categories (Major, Moderate, Minor) based on the highest impact magnitude they have reached. They were further ranked within these three categories, from the species with the higher impact risk to the species with the lower impact risk. $95 \%$ Confidence intervals given along the impact risk indicate that we can be $95 \%$ confident that the impact risk of a species lies within the upper and lower bounds (in squared brackets), and can therefore be considered as indicators of the uncertainty about each impact risk (the wider the interval, the higher the uncertainty). Impact risks were calculated from the species' frequencies of highest impact, which are the numbers of regions in which the alien species caused their highest impact magnitudes (on their total number of regions with impact observations).

\begin{tabular}{|c|c|c|c|c|c|c|c|}
\hline Alien scientific name(s) & Common name & Order & Family & $\begin{array}{l}\text { Highest impact } \\
\text { magnitude }\end{array}$ & $\begin{array}{l}\text { Frequency } \\
\text { of highest } \\
\text { impact }\end{array}$ & Impact risk & Rank \\
\hline $\begin{array}{l}\text { Ovis orientalis (syn. Ovis aries } \\
\text { musimon, Ovis ammon } \\
\text { musimon) }\end{array}$ & Mouflon & Cetartiodactyla & Bovidae & Major (MR) & $1 / 1$ & $\begin{array}{c}0.67[0.22 \\
1.00]\end{array}$ & 1 \\
\hline Camelus dromedarius & Dromedary & Cetartiodactyla & Camelidae & Major (MR) & $1 / 2$ & $\begin{array}{c}0.50[0.09 \\
0.91]\end{array}$ & 2 \\
\hline $\begin{array}{l}\text { Rusa timorensis (syn. Cervus } \\
\text { timorensis) }\end{array}$ & Javan deer & Cetartiodactyla & Cervidae & Major (MR) & $1 / 3$ & $\begin{array}{c}0.40[0.04 \\
0.77]\end{array}$ & $3-4$ \\
\hline Odocoileus virginianus & White-tailed deer & Cetartiodactyla & Cervidae & Major (MR) & $1 / 3$ & $\begin{array}{c}0.40[0.04 \\
0.77]\end{array}$ & $3-4$ \\
\hline Ovis aries & Sheep & Cetartiodactyla & Bovidae & Major (MR) & $1 / 5$ & $\begin{array}{l}0.29[0.02 \\
0.59]\end{array}$ & 5 \\
\hline Bos taurus & Cattle & Cetartiodactyla & Bovidae & Major (MR) & $1 / 7$ & $\begin{array}{l}0.22[0.01 \\
0.48]\end{array}$ & 6 \\
\hline Cervus nippon & Sika deer & Cetartiodactyla & Cervidae & Major (MR) & $4 / 23$ & $\begin{array}{c}0.20[0.06 \\
0.35]\end{array}$ & 7 \\
\hline Capra hircus & Goat & Cetartiodactyla & Bovidae & Major (MR) & $1 / 19$ & $\begin{array}{l}0.10[0.00 \\
0.22]\end{array}$ & 8 \\
\hline Bubalus bubalis & Water buffalo & Cetartiodactyla & Bovidae & Moderate (MO) & $3 / 3$ & $\begin{array}{c}0.80[0.47 \\
1.00]\end{array}$ & $9-10$ \\
\hline Axis axis & Chital & Cetartiodactyla & Cervidae & Moderate (MO) & $3 / 3$ & $\begin{array}{c}0.80[0.47 \\
1.00]\end{array}$ & $9-10$ \\
\hline Rangifer tarandus & Reindeer & Cetartiodactyla & Cervidae & Moderate (MO) & $2 / 2$ & $\begin{array}{c}0.75[0.37 ; \\
1.00]\end{array}$ & 11 \\
\hline Cervus elaphus & Red deer & Cetartiodactyla & Cervidae & Moderate (MO) & $4 / 5$ & $\begin{array}{l}0.71[0.41 \\
0.98]\end{array}$ & 12 \\
\hline Hemitragus jemlahicus & Himalayan tahr & Cetartiodactyla & Bovidae & Moderate (MO) & $1 / 1$ & $\begin{array}{c}0.67[0.22 \\
1.00]\end{array}$ & $\begin{array}{c}13-14-15- \\
16\end{array}$ \\
\hline Elephas maximus & Asian elephant & Proboscidea & Elephantidae & Moderate (MO) & $1 / 1$ & $\begin{array}{c}0.67[0.22 \\
1.00]\end{array}$ & $\begin{array}{c}13-14-15- \\
16\end{array}$ \\
\hline Oryx gazella & Gemsbok & Cetartiodactyla & Bovidae & Moderate (MO) & $1 / 1$ & $\begin{array}{c}0.67[0.22 \\
1.00]\end{array}$ & $\begin{array}{c}13-14-15- \\
16\end{array}$ \\
\hline Lama guanicoe & Guanaco & Cetartiodactyla & Camelidae & Moderate (MO) & $1 / 1$ & $\begin{array}{c}0.67[0.22 \\
1.00]\end{array}$ & $\begin{array}{c}13-14-15- \\
16\end{array}$ \\
\hline Dama dama & Fallow deer & Cetartiodactyla & Cervidae & Moderate (MO) & $8 / 12$ & $\begin{array}{l}0.64[0.40 \\
0.87]\end{array}$ & 17 \\
\hline Equus caballus & Horse & Perissodactyla & Equidae & Moderate (MO) & $8 / 14$ & $\begin{array}{c}0.56[0.33 \\
0.79]\end{array}$ & 18 \\
\hline
\end{tabular}




\begin{tabular}{|c|c|c|c|c|c|c|c|}
\hline Muntiacus reevesi & Reeves' muntjac & Cetartiodactyla & Cervidae & Moderate (MO) & $4 / 7$ & $\begin{array}{c}0.56[0.25 \\
0.85]\end{array}$ & 19 \\
\hline Oreamnos americanus & Mountain goat & Cetartiodactyla & Bovidae & Moderate (MO) & $2 / 4$ & $\begin{array}{c}0.50[0.15 \\
0.85]\end{array}$ & 20 \\
\hline Odocoileus hemionus & Mule deer & Cetartiodactyla & Cervidae & Moderate (MO) & $1 / 2$ & $\begin{array}{c}0.50[0.09 \\
0.91]\end{array}$ & $\begin{array}{c}21-22-23- \\
24\end{array}$ \\
\hline Ammotragus lervia & Aoudad & Cetartiodactyla & Bovidae & Moderate (MO) & $1 / 2$ & $\begin{array}{c}0.50[0.09 \\
0.91]\end{array}$ & $\begin{array}{c}21-22-23- \\
24\end{array}$ \\
\hline Cervus canadensis & Wapiti & Cetartiodactyla & Cervidae & Moderate (MO) & $1 / 2$ & $\begin{array}{c}0.50[0.09 \\
0.91]\end{array}$ & $\begin{array}{c}21-22-23- \\
24\end{array}$ \\
\hline $\begin{array}{l}\text { Rusa unicolor (syn. Cervus } \\
\text { unicolor) }\end{array}$ & Sambar deer & Cetartiodactyla & Cervidae & Moderate (MO) & $1 / 2$ & $\begin{array}{c}0.50[0.09 \\
0.91]\end{array}$ & $\begin{array}{c}21-22-23- \\
24\end{array}$ \\
\hline Sus scrofa & Wild boar & Cetartiodactyla & Suidae & Moderate (MO) & $11 / 23$ & $\begin{array}{c}0.48[0.29 \\
0.67]\end{array}$ & 25 \\
\hline Equus asinus & Donkey & Perissodactyla & Equidae & Moderate (MO) & $1 / 5$ & $\begin{array}{c}0.29[0.02 \\
0.59]\end{array}$ & 26 \\
\hline Bison bison & American bison & Cetartiodactyla & Bovidae & Minor (MN) & $1 / 1$ & $\begin{array}{c}0.67[0.22 \\
1.00]\end{array}$ & 27 \\
\hline
\end{tabular}


Table 3. Contingency table of the EICAT classifications of alien ungulates, amphibians (Kumschick et al., 2017), birds (Evans et al., 2016) and bamboos (Canavan et al., 2019): number of species assigned to different impact magnitudes.

\begin{tabular}{|c|c|c|c|c|c|c|}
\hline \multicolumn{2}{|c|}{ Impact magnitude } & Alien ungulates & Alien amphibians & Alien birds & Alien bamboos & Total \\
\hline \multirow{3}{*}{ 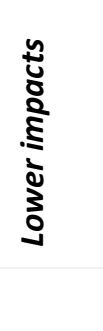 } & Minimal Concern (MC) & 0 & 4 & 36 & 0 & 40 \\
\hline & Minor (MN) & 1 & 20 & 46 & 2 & 69 \\
\hline & & 1 & 24 & 82 & 2 & 109 \\
\hline \multirow{5}{*}{ 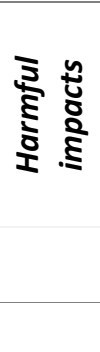 } & Moderate (MO) & 18 & 7 & 28 & 2 & 55 \\
\hline & Major (MR) & 8 & 5 & 4 & 6 & 23 \\
\hline & Massive (MV) & 0 & 4 & 5 & 0 & 9 \\
\hline & & 26 & 16 & 37 & 8 & 87 \\
\hline & Total & 27 & 40 & 119 & 10 & 196 \\
\hline
\end{tabular}




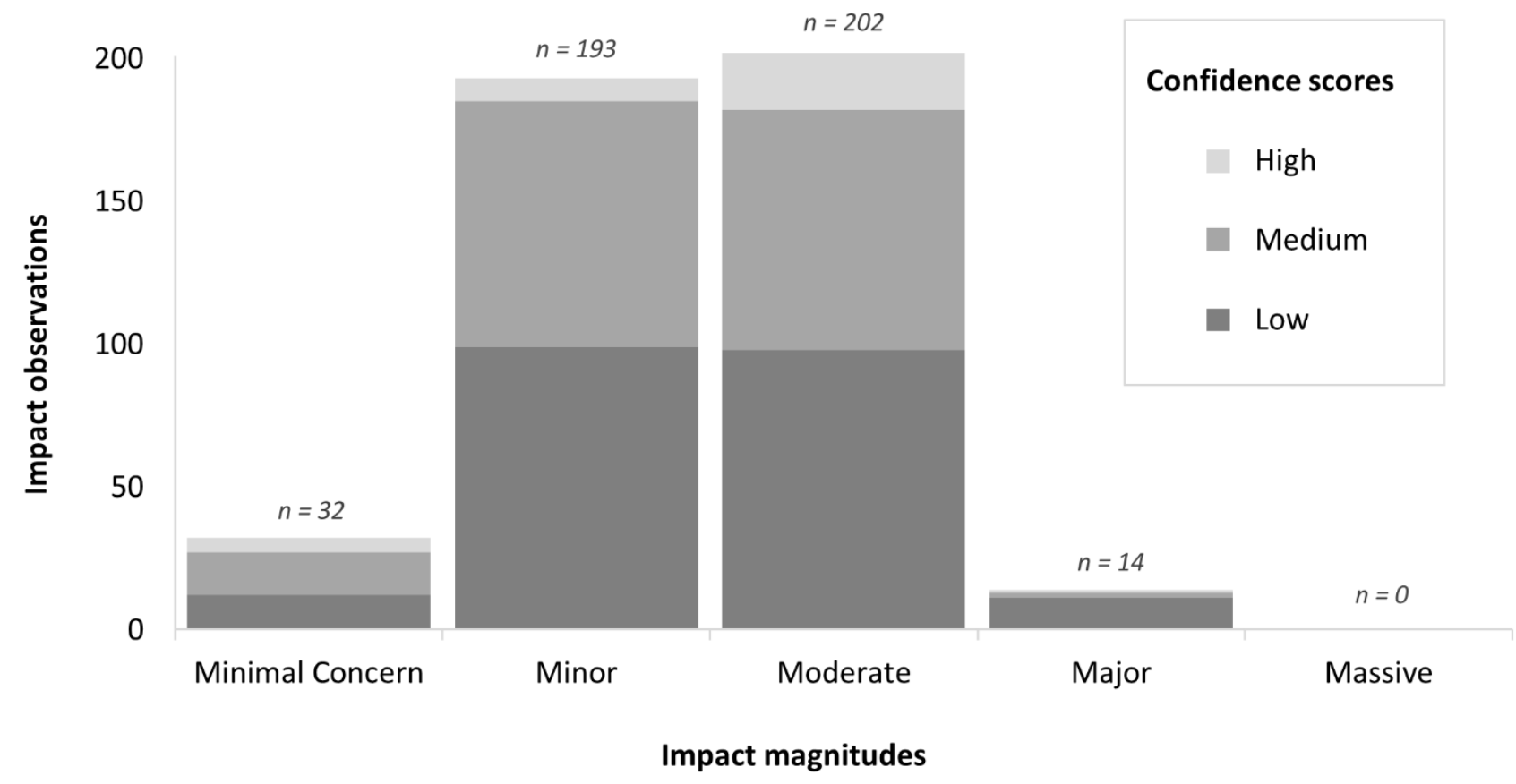

Figure 1. Impact magnitude (and their confidence scores) distribution of all impact observations of alien ungulates classified with EICAT $(\mathrm{N}=441)$. Within each impact magnitude, the lighter shade (top part) represents observations classified with a low confidence score, the intermediate shade (middle part) represents observations classified with a medium confidence score, and the darker shade (bottom part) represents observations classified with a high confidence score. 


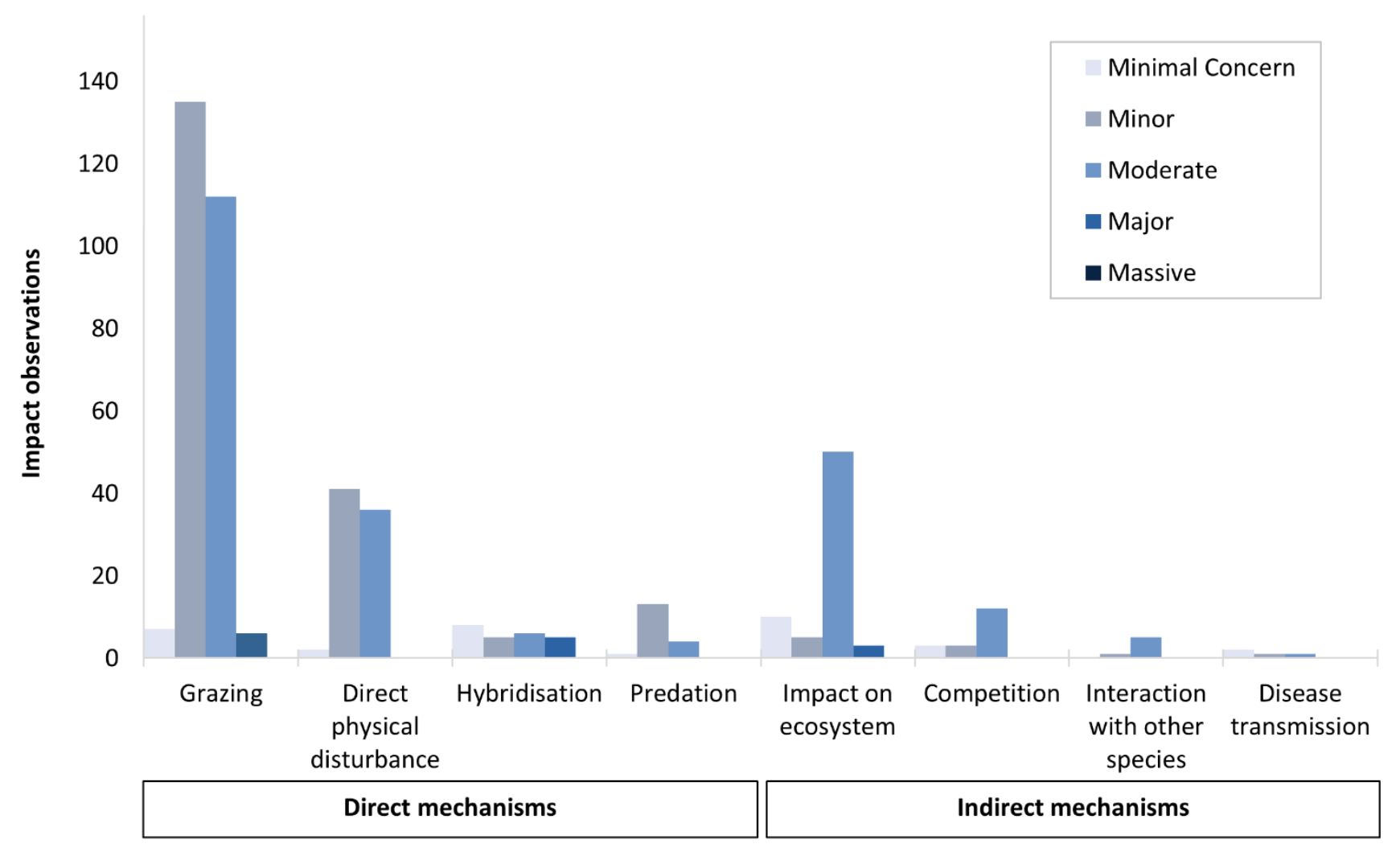

Figure 2. Impact mechanism distribution of all impact observations of alien ungulates classified with EICAT. Impact mechanisms can be grouped into direct and indirect mechanisms. Within each impact mechanism, the different blue shades represent observations classified into the five impact magnitudes. No Massive impacts were recorded for any species, under any of the mechanisms. Impact mechanisms can be classified as direct (i.e. alien taxon directly interacts with the impacted native taxon), or indirect (i.e. alien taxon modifies another factor of the environment, thereby indirectly affecting the native taxon). 


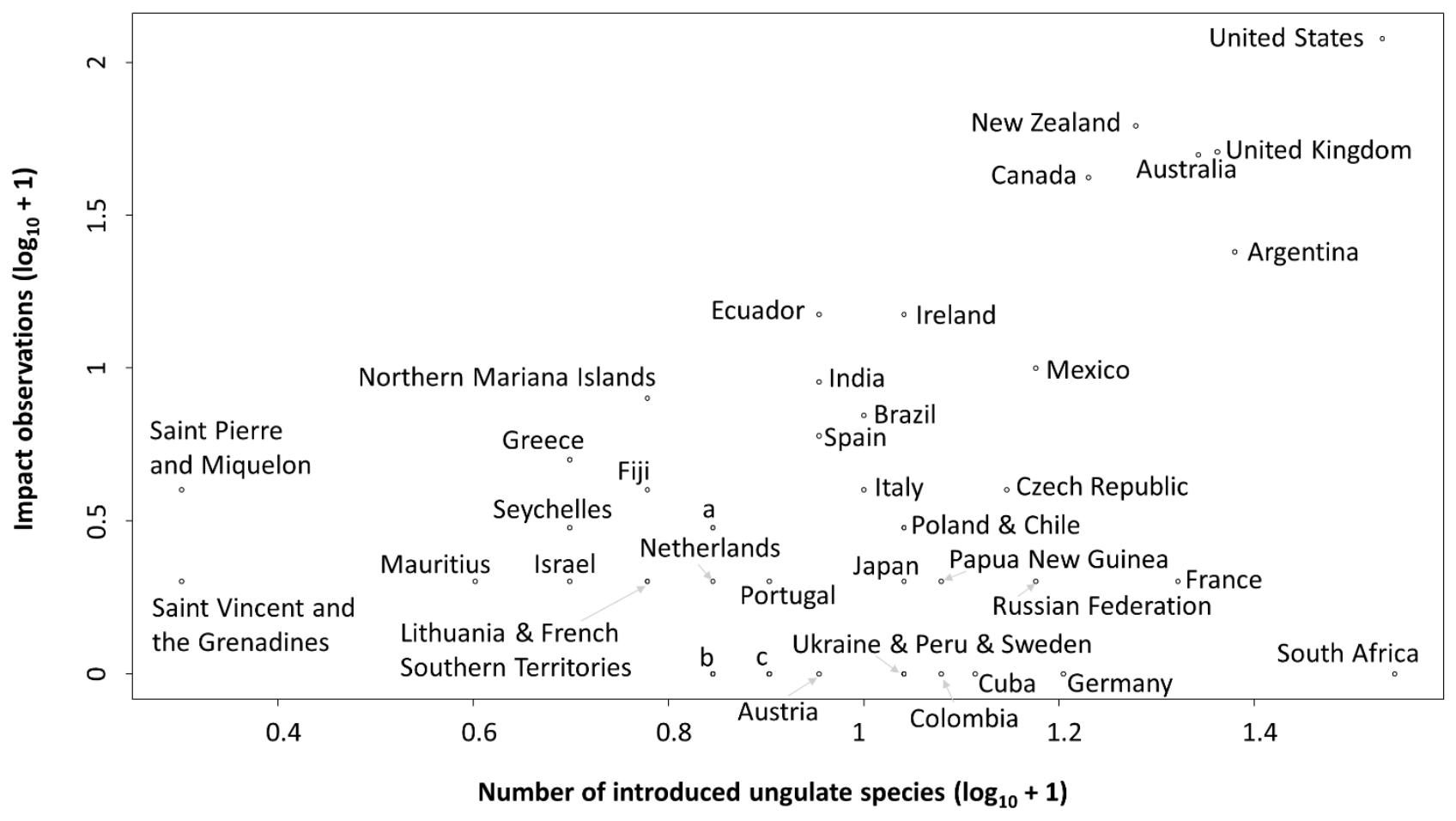

Figure 3. Relationship between the number of impact observations found per country, and the number of introduced ungulate species per country (see Sheet 3 in Supp. 2). Countries towards the top right are well-studied countries with many introduced ungulate species; countries towards the bottom right are countries with many introduced ungulate species but comparatively few impact observations. The letter a. stands for New Caledonia and the Falkland Islands, the letter b. stands for Bolivia, Denmark, Finland, Grenada, Haiti, Indonesia, Kazakhstan, Latvia, Madagascar, Saint Kitts and Nevis, Sao Tomé and Principe, Slovenia, Sri Lanka, Switzerland, Vanuatu, Venezuela and Yemen, and the letter c. stands for Antigua and Barbuda, Belgium, China, Kyrgyzstan, Malaysia and Slovakia. 


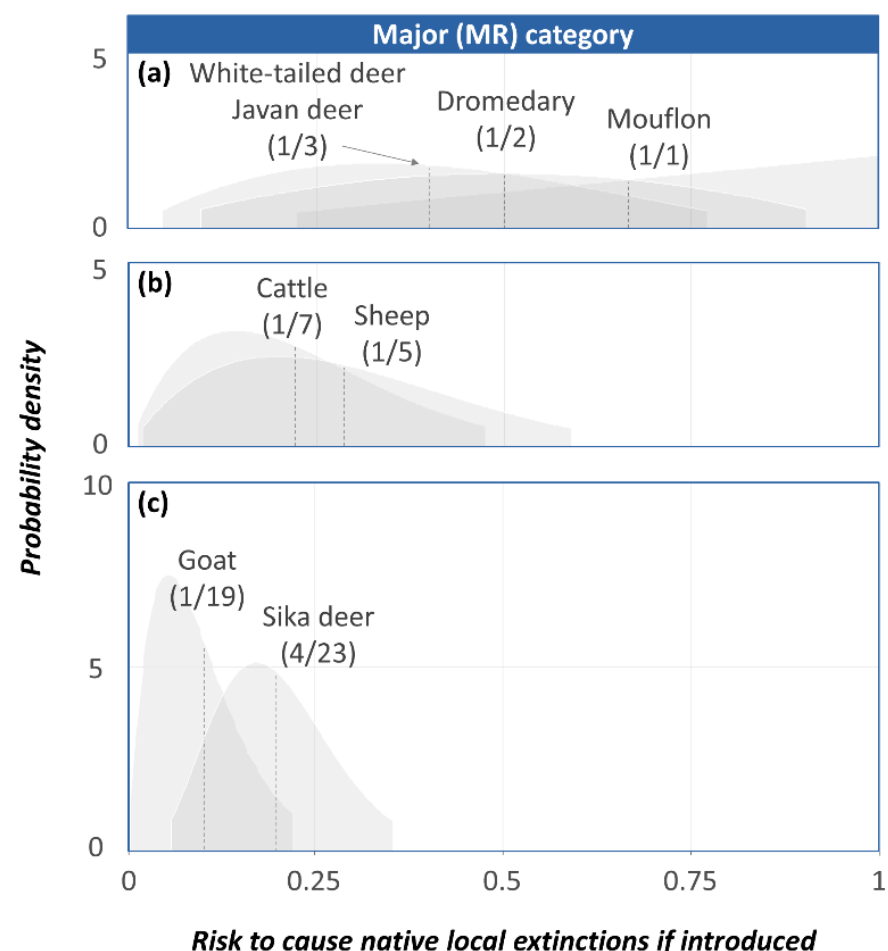

Risk to cause native local extinctions if introduced
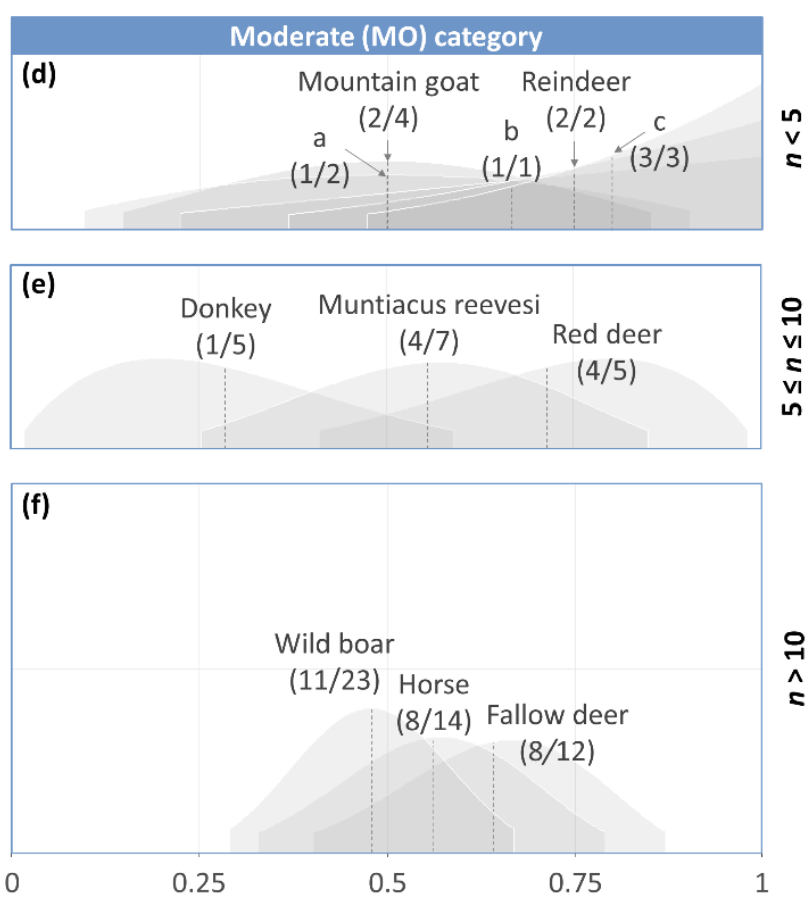

Risk to cause native population declines if introduced

Figure 4. Impact risk of the 26 alien species classified in the Major (left column; i.e. species whose alien populations have led to at least one documented local extinction of a native species) or Moderate (right column; i.e. species whose alien populations have led to at least one decline in a native population) categories. Impact risk is represented by the mean (vertical dashed lines), with beta density probability distributions providing $95 \%$ confidence intervals. Frequencies given after the species names indicate the numbers of regions (i.e. countries' sub-units) in which each alien species reached its highest impact magnitude/on the total number of regions with impact observations. For better visualisation, the species have been split into three groups: (a) and (d) show species with wider confidence intervals and high uncertainty in their risk of causing their highest impact magnitude $(<5$ regions with impact observations [n]); (b) and (e) show species with intermediate confidence interval widths and medium uncertainty in their risk of causing their highest impact magnitude $(5 \leq n \geq 10)$; and (c) and (f) show species with narrow confidence intervals and lower uncertainty in their risk of causing their highest impact magnitude $(n>10)$. In (e), the letter a. stands for the mule deer, aoudad, wapiti and sambar deer, the letter b. stands for the Himalayan tahr, Asian elephant, gemsbok and guanaco, and the letter c. stands for the chital and water buffalo. 